\title{
Coherent Backscatter Enhances Reflection Confocal Microscopy
}

BioTechniques 25:858-866 (November 1998)

\author{
Charles H. Keith, Glenn J. \\ Bird and Mark A. Farmer \\ University of Georgia, Athens, \\ GA, USA
}

\section{INTRODUCTION}

Transmission-mode differential interference contrast (DIC) microscopy and its electronically enhanced version, video-enhanced DIC microscopy, are the techniques most commonly used for studies of intracellular vesicle trafficking and cytoskeletal disposition in isolated living cells growing on transparent substrates. The images produced by these techniques have resolutions approaching the theoretical limit for light microscopy, are not contaminated by halos and have good optical sectioning characteristics $(13,22)$. Unfortunately, on opaque substrates, these techniques cannot be used. Although reflectionmode DIC microscopy has been described for metallographic substrates and for biological specimens stained with colloidal gold (3), it has not been widely used in biology. Reflection-contrast microscopy is a widely used technique, both in the standard epi-mode $(7,13,18,19)$ and in the confocal mode $(4,5,10,11,15)$, and when presented with specimens either of high reflectance (albedo) or that have substantial changes in index-of-refraction at boundaries (7), it can produce very high-resolution images. A new and exciting technique, aperture-scanning, reflected-light microscopy (6), can take fuller advantage of the full aperture of the objective and offer higher lateral and $\mathrm{z}$-axis resolution than reflection-contrast microscopy. However, most living, unstained biological substrates do not have sufficient albedo or difference in index-of-refraction to produce high-quality images by reflection-contrast microscopy techniques. Notable exceptions to this generalization are seen in the cornea (15) and in isolated cells migrating in three-dimensional collagen gels $(11,16)$ in which both the surface of the cells and the collagen fibrils can be visualized at a high resolution $(10,11)$. In both of these cases, the tissue, or the surrounding medium, is sufficiently transparent that the cell boundaries can be imaged in reflection confocal microscopy. This technique does not appear to image intracellular organelles as effectively as the plasma membrane $(11,15)$.

We have found that cells grown on reflective substrates produce an image similar to a DIC image when imaged with low numerical aperture (NA) objectives in the reflection mode of the confocal laser-scanning microscopy (CLSM). Both intracellular organelles and filopodia are visualized in these images to at least the same extent as in transmission DIC images with equivalent objectives. However, unlike transmission DIC, this technique can be used on opaque substrates. This visualization suggests that this technique can be useful for studies of organelle trafficking and axonal pathfinding on substrates in which traditional methods for visualizing live cells have proved difficult. The technique is simple to use, and the analysis of the mode of contrast formation could lead to to new modes of confocal microscopy. 


\section{MATERIALS AND METHODS}

PC12 cells were obtained from ATCC (Rockville, MD, USA). They were cultured on rat-tail collagen (Sigma Chemical, St. Louis, MO, USA) in Roswell Park Memorial Institute Medium 1640 (Grand Island Biological Company, Grand Island, NY, USA), supplemented with $10 \%$ horse serum (Hazleton Research Products, Denver, PA, USA) and 5\% fetal calf serum (FCS) (Life Technologies, Gaithersburg, MD, USA). For at least seven days before an experiment, they were cultured in the above medium with $4 \%$ horse serum and 2\% FCS, supplemented with $100 \mathrm{ng} / \mathrm{mL}$ of $2.5 \mathrm{~S}$ Nerve Growth Factor (Collaborative Biological Products, Bedford, MA, USA). One day before an experiment, they were plated on mirrored substrates (described below) coated with polyethyleneimine (PEI). For experiments in which nonimmersion or water-immersion objectives were used, cells fixed in $1 \%$ glutaraldehyde (Polysciences, Warrington, PA, USA) were imaged in phosphatebuffered saline (PBS). For experiments in which oil-immersion objectives were used, cells were fixed in $1 \%$ glutaraldehyde, immersed in mounting medium (Gel/Mount ${ }^{\mathrm{TM}}$; Fisher Scientific, Pittsburgh, PA, USA) and overlaid with a cover glass before being visualized.

Mirrored substrates were made by evaporating gold wire onto a $18-\mathrm{mm}^{2}$ coverslip to approximately $0.5-1.0$ optical density (OD) (assessed visually), using a Denton Vacuum Evaporator DV-502A (Denton Instruments, Cherry Hill, NJ, USA). These coverslips were coated with PEI (14) and were placed into a $35-\mathrm{mm}^{2}$ tissue culture dish for cell growth and a $60-\mathrm{mm}^{2}$ tissue culture dish for microscopy.

Confocal laser-scanning microscopy was done in reflection mode with a Model MRC-600 CLSM head (BioRad, Hercules, CA, USA) on a Nikon UV-PHOT microscope (Nikon, Melville, NY, USA), using a $1 / 4 \lambda$ plate (Bio-Rad) to suppress reflection artifacts from the lens surfaces (5). The CLSM head was equipped with a Krypton-Argon laser. The exciting illumination was generally restricted to $488 \mathrm{~nm}$ by adding a narrow-band interference filter on the excitation side of the re- flection filter set and was attenuated with the ND 2 filter in the excitation path of the microscope. The microscope was critically focused on the filopodia at the leading edge of the growth cone. Image brightness and contrast were adjusted using the black level and gain controls of the detection photomultiplier. Both water- and oilimmersion and non-immersion microscope objectives were used [non-immersion: Fluor $20 \times(0.75 \mathrm{NA}), 40 \times \mathrm{DL}$ (0.55 NA) (both from Nikon); water immersion: CF-WI 40× (0.55 NA) (Nikon), Plan NeoFluar ${ }^{\mathrm{TM}} 25 \times(0.81$ NA), Plan NeoFluar 40× (0.91 NA) (both from Carl Zeiss, Thornwood, NY, USA); oil immersion: Fluor $40 \times$ (1.33/0.8 NA), PlanApo 60× (1.4 NA) and Plan $100 \times(1.25 \mathrm{NA})$ (all from Nikon)]. The final on-screen magnification of the images was adjusted to fill the image with the object-of-interest with the scan area adjustment of the microscope control software (CoMOS; Bio-Rad).

Because the background gray level of the confocal images was set manually, using the black level and gain controls of the scan head, there was some variation in average brightness of the resulting images. Also, there was some modest variation in brightness across

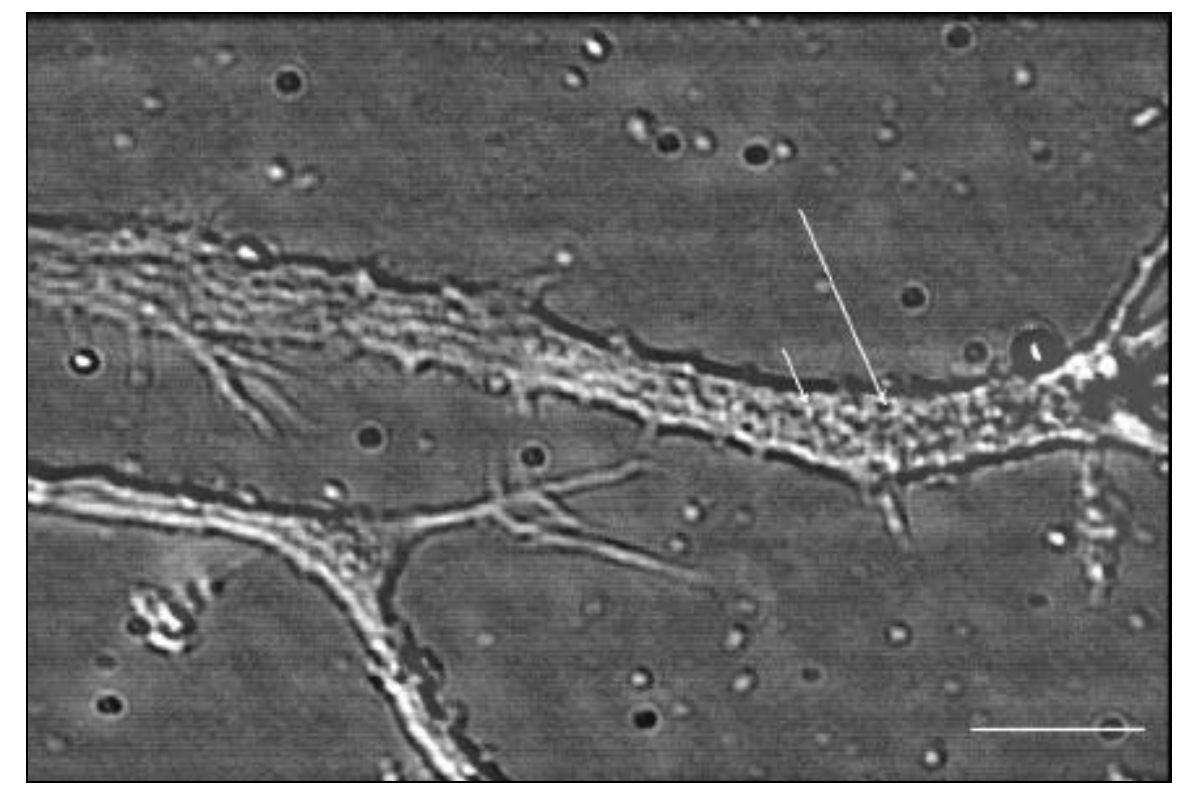

Figure 1. The shaft and filopdial extensions of PC12 neurites, grown on a reflective gold substrate, are readily visualized in backscatter-enhanced reflection confocal microscopy. Note that the vesicles are visualized either as pits (long arrow) or lands (short arrow), and the linear structures, probably bundles of microtubules, are well visualized within the neurite shaft. Bar $=10 \mu \mathrm{m}$.

many of the images. These variations were equalized within individual images and between images using the Image-Pro Plus ${ }^{\mathrm{TM}}$ Image Processing Program (Media Cybernetics, Silver Spring, MD, USA). Confocal images were translated into tagged image file format (TIFF) using the public domain program Confocal Assistant (Todd Clarke Belje, University of Minnesota Medical Center). They were then transferred into Image-Pro Plus to be put onto a uniform gray scale. Background was flattened across each image using a flattening filter with a feature width of 20 pixels, and image brightness was adjusted to give a uniform average background gray level in all images.

\section{RESULTS}

Reflection CLSM of fixed PC12 neurites on mirrored substrates (Figure 1) results in images that are reminiscent of transmission DIC images of neurites grown on transparent substrates (1). Filopodia at the leading edge of the growth cone are well visualized (Figure 1 and Figure 2A). Vesicular structures within the growth cone cytoplasm are visualized either as small indentations (pits) or extrusions (lands), and linear 
structures reminiscent of the structures that have been identified as microtubules in DIC images can barely be perceived (Figure 1).

The contrast and apparent resolution "quality" of reflection CLSM images was optimized with water-immersion lenses of the lowest available NA. The Nikon CF-WI 40× (0.55 NA) gave the best images, followed closely by the Zeiss 25× Plan-NeoFluar (0.81 NA) water-immersion objective. The use of higher magnification, higher NA wateror oil-immersion objectives [Zeiss 40X Plan-Neofluar (0.91 NA), Nikon Fluor 40× (1.33 NA), Nikon PlanApo 60× (1.4 NA) and a Nikon Plan 100× (1.25 NA)] resulted in lower image quality (Figure 2). In part, this reduction in apparent image quality probably is the result of the depth of field being lower with high NA objectives. However, even in critically focused parts of the image (i.e., the juncture of the filopodia in Figure 2), the detail available is less with high NA objectives. Additionally, the use of non-immersion objectives of slightly lower [Nikon Fluor 20× $(0.75$ NA)] or equal [Nikon $40 \times$ DL $(0.55$ NA)] NA also resulted in lower image quality (data not shown.) It is not clear whether the degradation seen with nonimmersion objectives was due to the change in objective NA or due to reflection effects at the glass-air and airwater interfaces.

These images were dependent on the presence of a reflective substrate and on the close apposition between the reflective substrate and the object to be imaged. While cells can be visualized in reflection CLSM on non-reflective substrates, the contrast of intracellular features is rather low in such images $(11,15)$. We found that the most reflective substrates (ca. 1 OD coating) gave better images than substrates coated with a lighter layer of gold (ca. 0.5 OD coating), and that uncoated substrates or substrates coated on the opposite side of the coverslip, gave the poorest images of intracellular features (Figure 3).

The quality of reflection CLSM images was also critically dependent on the size of the confocal aperture. Adjustment of the pinhole to the minimum aperture available (ca. $50 \mu \mathrm{m}$ ) gave optimal image quality; opening the aperture even slightly (to ca. $100 \mu \mathrm{m}$ ) dra- matically degraded images, even when the effects of increased signal were accounted for by adjustment of amplifier black level and gain (data not shown).

\section{CONCLUSIONS}

\section{Mode of Contrast Enhancement}

Any model for the mode of contrast enhancement in these images must take into account the observations listed in Table 1.

The described dependence of image-contrast enhancement on close ap-
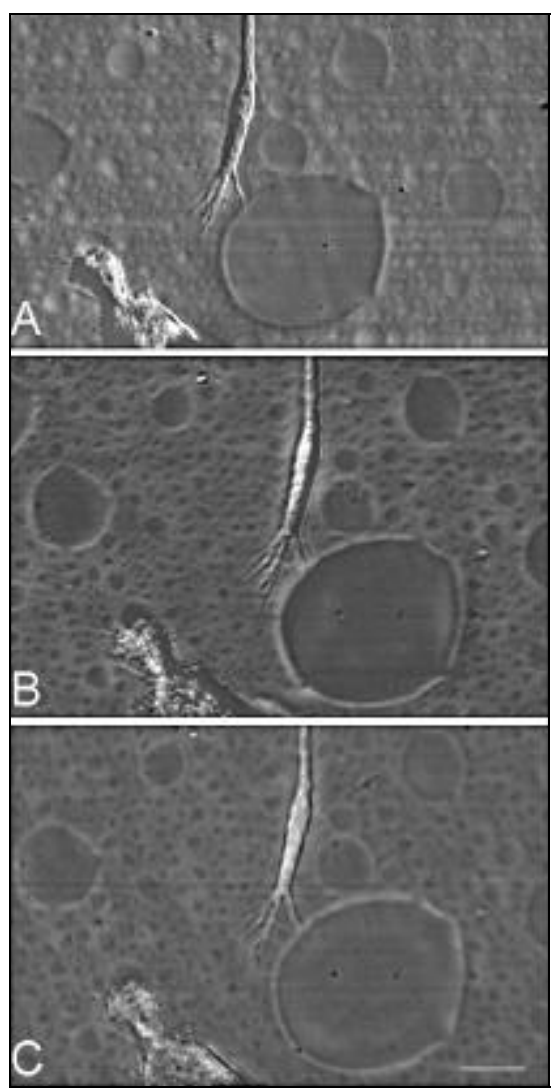

Figure 2. The contrast of features visualized in PC12 neurites by backscatter-enhanced reflection confocal microscopy is optimized with low NA objectives. The visualization of the filopodia and intraneuritic details of PC12 neurites on reflective substrates is highest when a $40 \times, 0.55$ NA Nikon CF-WI objective is used (A). When a higher NA objective, such as a $25 \times, 0.81-N A$ Zeiss Plan Neofluar (B) or a 40×, 0.91-NA Zeiss Plan Neofluar (C) is used, visualization of features is poorer. (Note that the circular features in the substrate are microdroplets of PEI. These are seen when coverslips are stored for extensive periods of time before plating cells. They do not appear to affect either cell growth or visualization of cellular features.) $\mathrm{Bar}=10 \mu \mathrm{m}$. position between scattering object and reflective surface implies that contrast enhancement depends on interference between incoming laser illumination scattered from the specimen and backscattered illumination reflected off the mirrored substrate. (Figure 4). Because laser light is coherent, as long as the mirrored substrate is reasonably close to the specimen, there will be a phase shift between scattered and backscattered light that will depend on the separation between object and mirror. At larger cell-substrate separations, this relationship breaks down because the convergence of incoming illumination introduces dispersion in the phase of the backscattered light. This dispersion in phase is more extreme for offaxis illumination, so low NA lenses, in which the cone of illumination is closely paraxial, give better contrast
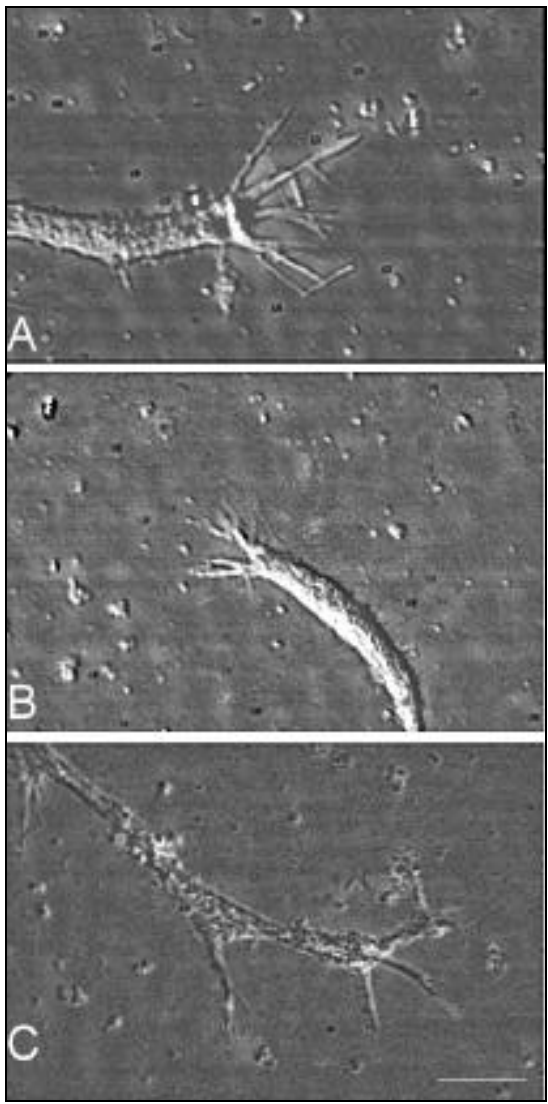

Figure 3. The contrast of features visualized in backscatter-enhanced reflection confocal microscopy is optimized by more reflective substrates. The visualization of filopodia and intraneuritic vesicles is better when cells are grown on a thicker gold substrate (ca. 1.0 OD; A) than on a thinner gold substrate (ca. 0.5 OD; B) or on an uncoated coverslip (C). Bar $=10 \mu \mathrm{m}$. 


\section{Contrast enhancement is dependent on having a highly reflective substrate} closely apposed to the object of interest.

2. Image quality is degraded by increasing the size of the confocal aperture.

3. Vesicles are visualized either as pits or lands, depending, presumably, on their height from the substrate.

4. Image quality is better with low NA objectives.

5. Images are better with monochromatic than with polychromatic illumination.

enhancement than high NA lenses. Visualization requires a small confocal aperture to separate the signal from the cell from the backscattered illumination. Presumably, polychromatic illumination degrades signal quality more than in reflection confocal (10) because microscope objectives are not corrected for chromatic abberation away from their usual focal point.

The above contrast is substantially enhanced by the electronic characteristics of the light-detection system, as described for video-enhanced polarization and DIC microscopy $(2,12,13)$. Because the detector-in this case, a photomultiplier tube-shows a linear response to light, the black level control of the detector electronics can be used to subtract a DC offset from the signal, leaving an enhanced scattering signal.

If contrast is occurring because of interference between backscattered illumination and scattering from features in the cell, it is to be expected that a linear object that is oriented in a direction other than horizontal or vertical should show interference fringes along its length. Unfortunately, the filopodia extending out from the growth cones in out images, many of which appear to extend at an angle, do not show obvious

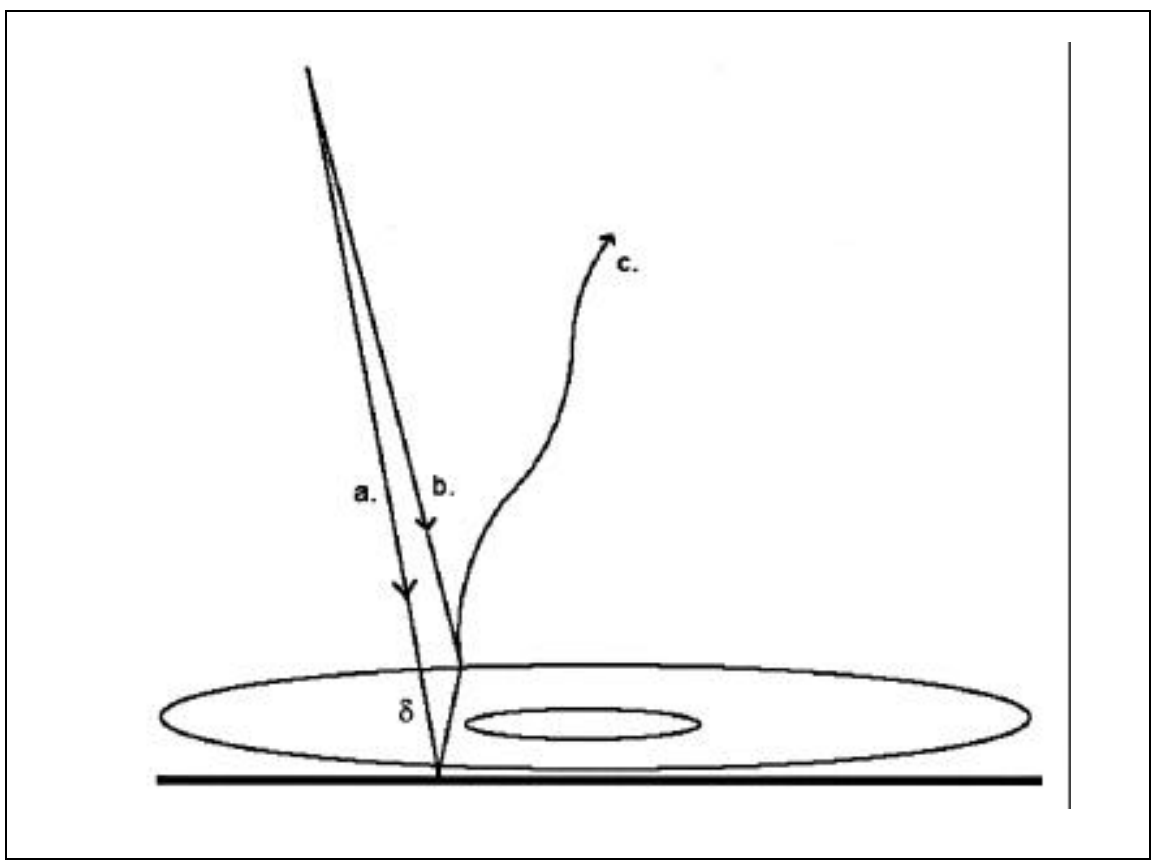

Figure 4. Proposed mode of contrast generation in backscatter-enhanced reflection confocal microscopy. The incoming laser illumination (rays a and b) is coherent. Scattering of ray a at the cell surface induces a phase shift in that component; additionally, the increase in optical path $(2 \times \delta)$ for ray $b$ introduces a phase shift in that component. Interference between the two components gives rise to interference contrast in the resultant ray c. Ray c can be seen relative to the much brighter specularly reflected ray b, because ray b is not in focus at the confocal aperture and is largely blocked. 
fringes. We think that these fringes are not seen for two reasons: (i) Typical filopodia from a cultured neuron are approximately $0.2 \mu \mathrm{m}$ in thickness; 0.2 $\mu \mathrm{m}$ is a significant fraction of the wavelength of the light used, which will tend to make fringes less visible. (ii) The convergence of the illumination induces phase dispersion in the backscattered illumination, which reduces the sharpness of any fringes.

\section{Other Techniques}

This new technique of backscatterenhanced reflection confocal microscopy has a great deal in common with three other modes of microscopy: (i) reflection contrast microscopy (including reflection confocal microscopy), (iii) interference reflection contrast microscopy (including interference reflection confocal microscopy) and (iii) dual-pass confocal micro- scopy. In reflection contrast microscopy, plane polarized light illuminates the specimen, and the reflected signal is viewed through a polarizer at $90^{\circ}$ to the illuminating polarizer, eliminating scatter from other interfaces (18); in the confocal microscope, the confocal aperture eliminates the need for the polarizers (5). Contrast appears to be proportional to the difference of the squares of the refractive index of the scattering object relative to the surrounding medium $(5,7)$. Since there are significant differences between the refractive index of cells and surrounding medium, this technique gives good visualization of the surface but limited visualization of the intracellular features of unstained biological specimens (11). It also gives excellent contrast with peroxidase-stained specimens (7), immunogold-stained specimens (19) or collagen fibrils in aqueous medium (10). In interference reflection contrast microscopy, cells are generally made to adhere to a glass surface, which is placed closer to the objective than the cell. Destructive interference between the light reflected at the glass-medium interface and the medium-cell interface results in a reduction of the intensity of reflected light in regions of close apposition between the cell and coverslip $(8,17,23)$. In the type of microscopy we have described, the reflective surface is below the cell and is largely eliminated from the final image by the confocal aperture. The light reflected from it can, however, interfere with light scattered from regions of the cells away from the surface and with light scattered from multiple interfaces in the cytoplasm. Additionally, unlike our backscatter-enhanced technique, interference reflection contrast microscopy cannot be used with opaque surfaces, only with partially reflective surfaces such as a glass coverslip. 
A type of confocal microscopy with which this technique shares characteristics is double-pass transmission confocal microscopy $(9,20,21)$. In this technique, light transmitted through a specimen is focussed onto a planar or curved mirror. The reflected beam is then refocused onto the specimen, from whence it is imaged through the normal confocal reflection path and photodetector. This technique differs from ours in that a true transmission image is obtained, rather than an interference image. Furthermore, for obvious reasons, this technique cannot be used with opaque specimens.

We have been using backscatter-enhanced reflection confocal microscopy to visualize the disposition of the growth cone, and its organelles as elongating axons find a path along a patterned adhesive substrate; the reflection method is necessary because metal is shadowed over the substrate to modulate adhesivity. However, such a technique will be useful in other contexts such as studying the behavior of cells on substrates such as metal or silicon. Furthermore, if the issue of dispersion of the backscattered illumination can be addressed, the technique should be useable with high NA objectives and may give information about the interaction of living cells with opaque substrata that is difficult to obtain by other techniques.

\section{ACKNOWLEDGMENTS}

This work was supported under the auspices of the Center for Advanced Ultrastructural Research of the University of Georgia.

\section{REFERENCES}

1.Aletta, J.M. and L.A. Greene. 1988. Growth cone configuration and advance: a time-lapse study using video-enhanced differential interference contrast microscopy. J. Neurosci. 8:1425-1435

2.Allen, R.D., N.S. Allen and J.L. Travis. 1981. Video-enhanced contrast, differential interference contrast (AVEC-DIC) microscopy: a new method capable of analyzing microtubule-related motility in the reticulopodial network of Allegromia latticollaris. Cell Motil. 1:291-302.

3.Bajer A.S., H. Sato and J. Mole-Bajer. 1986. Video microscopy of colloidal gold particles and immuno-gold labelled microtubules in improved rectified DIC and epi-illumination. Cell Struct. Funct. 11:317-330.

4.Boyde, A. and S.J. Jones. 1995. Mapping and measuring surfaces using reflection confocal microscopy, p. 255-265. In J.B. Pawley (Ed.), Handbook of Biological Confocal Microscopy, 2nd ed. Plenum Press, New York.

5.Cheng, P.C. and A. Kriete. 1995. Image contrast in confocal light microscopy, p. 281-310. In J.B. Pawley (Ed.), Handbook of Biological Confocal Microscopy, 2nd ed. Plenum Press, New York.

6.Conchello, J.-A., J.P. Heym, J.L. Wei and J.W. Lichtman. 1997. Novel reflected light confocal profilometer. SPIE Proc. 2984:14.

7.Cornelese-ten Velde, I., J. Bonnet, H.J. Tanke and J.S. Ploem. 1988. Reflection contrast microscopy. Visualization of peroxidasegenerated diaminobenzidine polymer products and its underlying optical phenomena. Histochemistry 89:141-150.

8.Davies, P.F., A. Robotewskyj and M.L. Griem. 1993. Endothelial cell adhesion in real time. Measurements in vitro by tandem scanning confocal image analysis. J. Clin. Invest. 91:2640-2652.

9.Dixon, A.E. and C. Cogswell. 1995. Confocal microscopy with transmitted light, p. 479-490. In J.B. Pawley (Ed.), Handbook of Biological Confocal Microscopy, 2nd ed. Plenum Press, New York.

10.Friedl, P., K. Maaser, C.E. Klein, B. Niggemann, G. Krohne and K.S. Zänker. 1997.
Migration of highly aggressive MV3 melanoma cells in 3-dimensional collagen lattices results in local matrix reorganization and shedding of $\alpha 2$ and $\beta 1$ integrins and CD44. Cancer Res. 57:2061-2070.

11.Gunzer, M., E. Kampger, E.B. Brocker, K.S. Zanker and P. Friedl. 1997. Migration of dendritic cells in 3D-collagen lattices. Visualization of dynamic interactions with the substratum and the distribution of surface structures via a novel confocal reflection imaging technique. Adv. Exp. Med. Biol. 417:97-103.

12.Inoué, S. 1981. Video image processing greatly enhances contrast, quality, and speed in polarization-based microscopy. J. Cell Biol. 89:346-356.

13.Inoué, S. and K.R. Spring. 1997. Video Microscopy. The Fundamentals, Chapters 1 and 2. Plenum Press, New York.

14.Keith, C.H. and K. Blane. 1990. Sites of tubulin polymerization in PC12 cells. J. Neurochem. 54:1258-1268.

15.Masters, B.R. and M.A. Farmer. 1993. Three-dimensional confocal microscopy and visualization of the in situ cornea. Comput. Med. Imaging Graph. 17:211-219.

16.Niggemann, B., K. Maaser, H. Lu, R. Kroczek, K.S. Zanker and P. Friedl. 1997. Locomotory phenotypes of human tumor cells and $\mathrm{T}$ lymphocytes in a three-dimensional collagen lattice. Cancer Lett. 118:173-180.

17.Paddock, S.W. 1989. Tandem-scanning reflected-light microscopy of cell-substratum adhesions and stress fibres in Swiss 3T3 cells. J. Cell Sci. 93:143-146.

18.Ploem, J.S. 1975. Reflection-contrast microscopy as a tool for investigation of the attachment of living cells to a glass surface, $p$. 405-421. In R. van Furth (Ed.), Mononuclear Phagocytes in Immunity Infection and Pathology. Blackwell, Oxford.

19.Prins, F.A., R. van Diemen-Steenvoorde, J. Bonnet and I. Cornelese-ten Velde. 1993. Reflection contrast microscopy of ultrathin sections in immunocytochemical localization studies: a versatile technique bridging electron microscopy with light microscopy. Histochemistry 99:417-425.

20.Russ, J.C. 1995. The Image Processing Handbook, 2nd ed, p. 597-599. CRC Press, Boca Raton.

21.Sheppard, C.J.R. and T. Wilson. 1980. Multiple traversing of the object in the scanning microscope. Opt. Acta 27:611-624.

22.Slayter, E.M. and H.S. Slater. 1992. Light and Electron Microscopy, p. 149-167. Cambridge University Press, Cambridge, England.

23.Smith, C.L. 1994. Cytoskeletal movements and substrate interactions during initiation of neurite outgrowth by sympathetic neurons in vitro. J. Neurosci. 14:384-398

Received 6 April 1998; accepted 24 September 1998.
Address correspondence to:
Dr. Charles H. Keith
Department of Cellular Biology
University of Georgia
Athens, GA 30602, USA
Internet: chkeith@cb.uga.edu 